\title{
Research on Performance Test Characteristics of Solar Energy ORC Generator Set
}

\author{
Yongkang Zhang ${ }^{1}$, Jinghui Song ${ }^{2}$,Yunfeng $\mathrm{Xia}^{3}$ \\ 1Dongguan Guanneng Green Energy Service Co., Ltd. Dongguan 523000, China. \\ 2Guangdong Electric Power Research Institute Energy technologies Ltd. Guangzhou 510080, China. \\ 3 Guangdong Power Grid Co., Ltd. Dongguan Power Supply Bureau, Dongguan 523000, China.
}

\begin{abstract}
In order to study the performance of low-temperature solar-powered ORC generator sets, a solar-powered ORC power generation test bench was designed and built. In the experiment, R-123 was used as the organic Rankine cycle working fluid, and the solar ORC power generation system was experimentally studied. The research results show that when the direct solar radiation intensity is about $400 \mathrm{~W}$, the temperature of the heat transfer oil at the outlet of the collector can reach $140{ }^{\circ} \mathrm{C}$. When the temperature of the heat transfer oil at the outlet of the collector is around $110{ }^{\circ} \mathrm{C}$, the collector efficiency of the collector can reach about $60 \%$. Under the heat source condition, when the power cycle part is switched from the basic cycle to the regenerative cycle mode, the collector heat collection efficiency can reach about $60 \%$. Under the heat source condition, when the power cycle part is switched from the basic cycle mode to the regenerative cycle mode, the measured efficiency is increased from $9.3 \%$ to $10.8 \%$, and the measured cycle efficiency is increased from $1.57 \%$ to $1.67 \%$, which is an increase of $6.07 \%$. The measured cycle system efficiency is about $10 \%$, and the heat recovery mode is slightly higher than the basic cycle mode. The organic Rankine cycle performance under different working fluid flows was also investigated in the experiment. The maximum measured average power was $386.27 \mathrm{~W}$ when the working fluid flow was 6.88 $\mathrm{kg} \cdot \mathrm{s}$. At a certain heat source temperature, as the flow rate of the working fluid increases, the inlet pressure of the expander increases, and the circulating output work also increases. Under a certain working fluid flow rate, as the temperature of the heat source increases, the temperature of the inlet of the expander increases, and the inlet pressure increases. the cycle output work also increased.
\end{abstract}

\section{Introduction}

The organic Rankine cycle technology can perform good thermal work conversion on the heat source in the middle and low temperature range, and has better cycle performance than the water vapor Rankine cycle. It can be widely used in the field of medium and low temperature power generation, with good adaptability and economy. The heat source can be solar energy, geothermal energy, industrial waste heat waste heat or biomass energy, etc., and can effectively convert thermal energy into mechanical energy or electric energy in the range of 70-350 oC.

The research on the organic Rankine cycle mainly focuses on the selection of working fluids, theoretical analysis of cyclic performance, cyclic application analysis, and development of various expanders. In terms of working fluid selection, in addition to its good cycle performance, its stability, leakage, corrosivity, toxicity and non-flammability are some of the physicochemical properties in the selection of working fluids. . Hung et al. ${ }^{[1]}$ conducted a series of studies on applicable working fluids in different heat source temperature ranges in organic Rankine cycle systems, and selected the appropriate working fluids to achieve optimal system efficiency under suitable working conditions, pointing out that $\mathrm{R} 245 \mathrm{fa}$ is at $150^{\circ} \mathrm{C}$. Nishith et al ${ }^{[2]}$ studied the performance of 16 organic working fluids in the basic organic Rankine cycle and the optimized cycle, pointing out that dry working fluids and isentropic working fluids are more suitable for organic Rankine cycles. Manolakos et al. ${ }^{[3]}$ designed and built a low-temperature solar organic Rankine cycle system for reverse osmosis seawater desalination system in Greece, which uses R134a as the working medium and the working medium evaporates in the flat plate collector. The system performance was tested under the experimental conditions. The collector outlet temperature reached $77.3{ }^{\circ} \mathrm{C}$, the maximum output power of the system was $2.05 \mathrm{~kW}$, and the total system efficiency was $4 \%$. Nguyen et al. ${ }^{[4]}$ developed a system for generating electricity from a $100^{\circ} \mathrm{C}$ heat source, using n-pentane as the working fluid, producing $1.5 \mathrm{~kW}$ of electricity, and the system efficiency is $4.3 \%$. Christoph et al. ${ }^{[5]}$ used the Aspen software for the basic ORC cycle, regenerative cycle and saturation cycle with R245fa as the working fluid, and analyzed the economics of each cycle at different scales. Sotirios et 
al. ${ }^{[6]}$ conducted a multi-generation system coupled with vapor compression refrigeration using biomass and solar energy as a heat source, and also pointed out that regenerators can effectively improve performance. Wang

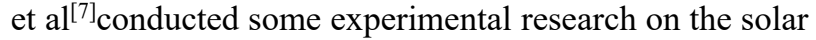
organic Rankine cycle system. The system used a plate collector as the collector evaporator, and used the throttle valve to simulate the expander. The experiment used R245fa as the circulating medium. The maximum experimental efficiency of the system is $3 \%$. Yan Gang et al.$^{[8]}$ carried out theoretical research and analysis on the solar-driven organic Rankine cycle system, and proposed to segment the endothermic process of the working fluid. The plate collector was used in the low temperature section and the CPC collector was used in the high temperature section. The device can achieve better cycle performance; and the heat transfer oil heater is used as the heat source, and the turboexpander is used as the power machine. The experiment is carried out under the temperature difference of $70^{\circ} \mathrm{C}$ cold and heat source, and the cycle efficiency reaches $6.8 \%$. Liu Linding ${ }^{[9]}$ designed a $10 \mathrm{~kW}$ organic Rankine cycle system with a single screw expander as the power machine, and mainly carried out experimental research on the performance of the single screw expander.

The above research work has important reference value for the promotion and application of the organic Rankine cycle system. The experimental systems in the existing research are relatively simple, most of them use hot water or electric heaters as heat sources for research, or use throttling devices instead of work devices for research, and there are few experimental studies of complete systems. Zhang Xiaosong et $\mathrm{a}^{\left[{ }^{[10]} \text { conducted a }\right.}$ preliminary study on the solar-driven organic Rankine cycle system, and carried out theoretical analysis and systematic development of the solar-driven organic Rankine cogeneration cycle system. Based on the previous research, the author designed and developed an experimental system, which used the trough solar collector to collect solar heating and heat transfer oil as the system heat source, and then exchanges the circulating refrigerant to drive the organic Rankine cycle system to realize the electric energy output and heat energy supply. The experimental system can control the switching between the basic ORC cycle, the regenerative cycle, and the cogeneration cycle. This paper introduces the experimental system, and mainly tests the heat collecting performance of the collector under medium solar radiation conditions and the actual cycle performance of the solar-driven organic Rankine cycle system to obtain the actual performance of the solar organic Rankine cycle system. The impact of each major parameter on the performance of the experimental system provides guidance and support for system design for large-scale operations.

\section{2 experimental system}

Fig.1 shows the flow chart of the experimental system. It mainly includes solar thermal steam generation system, ORC generator set and related auxiliary equipment such as valves and pipelines. The solar thermal steam generation system includes solar collectors and steam drums, cavity absorbers and circulating water pipes; ORC generator sets include heat exchangers, expanders, generators, oil storage tanks, condensers, refrigerant pumps, pulse dampers, oil pumps, hot water pipes, and related valves, pipes, etc. Auxiliary equipment; firstly, the water is passed through the solar collector to enter the steam drum for vapor-liquid separation, the liquid water is returned to the collector for heating again, and the steam is sent to the heat exchanger through the pipeline, and then the hot steam heats the organic working medium in the heat exchanger. Finally, the heated organic working fluid enters the expander to do work, the expander drives the generator to generate electricity, and the organic working fluid that has completed the work enters the condenser to complete the whole cycle, and the R-123 is used as the working medium in the power cycle in the experimental system.Table 1. Setting Word's margins.

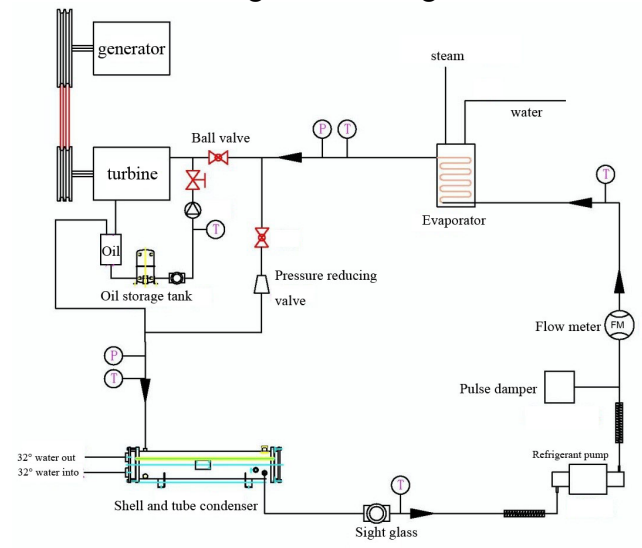

Fig. 1 Experimental system flow

\section{SYSTEM EVALUATION METHOD}

For the trough solar collector system, the input is mainly the direct radiant heat received on the reflective surface of the trough collector, and the output is the heat absorbed by the heat transfer oil collected in the system. The collected solar radiation heat absorbed by the heat transfer oil can be calculated from the collected direct radiation data and the parameters of the heat transfer oil temperature, flow rate, specific heat capacity, etc., so that the heat collection efficiency of the trough collector can be further obtained. For the organic Rankine cycle system, the main output is the output power of the screw expander and the electric energy generated by the generator. In the experiment, the collected temperature, pressure, flow and other data can be used to calculate the working fluid quality through the physical property software. The thermal parameters such as entropy, combined with the efficiency of the various components of the system, can obtain data such as heat absorption, heat recovery, heat release and work volume of the circulation system. The main evaluation indicators of the experimental system are the collector efficiency of the collector, the efficiency of the cycle work, and the total thermal efficiency of the system. 
The collector efficiency of the trough collector can be simplified as the ratio of the heat obtained by the heat transfer oil in the collector to the total direct radiant energy obtained on the mirror surface of the collector.

$$
\eta c=\frac{Q_{s c}}{A I_{b}}
$$

The cycle efficiency rate is the ratio of the net output power of the cycle (the difference between the output power of the screw expander and the power consumption of the working pump) and the heat absorption of the working medium in the steam generator.

$$
\eta_{t}=\frac{W_{E}-W_{P}}{Q_{V G}}
$$

Because of the difference in electrical energy and thermal energy taste, a single thermal efficiency does not clearly express the utilization of the cycle for low-grade energy. Therefore, the efficiency of the loop is introduced to evaluate the system. Efficiency is the ratio of the output power of the cycle to the input of the cycle. For the cycle, the output power is the output power WE of the expander, and the input yong is the sum of the heat input by the steam generator in the cycle Ein, vg and the power consumed in the working fluid pump by Wp, ie

$$
\eta_{E, c y c}=\frac{W_{E}}{E_{i n, V G}+W_{P}}
$$

Where, Qsc is the collector of the collector, A is the mirror area of the collector, $\mathrm{Ib}$ is the direct radiation intensity of the solar energy, QVG is the heat absorption of the working medium in the steam generator, is the output power of the expander, QR It is the heat exchange capacity of the working medium in the regenerator. Qc is the heat release amount of the working medium in the condenser, which is the amount of work energy of the working fluid pump.

\section{EXPERIMENTAL RESULTS AND ANALYSIS}

Fig. 2 and Fig. 3 show that the experimental results of the experimental system collected in the basic cycle mode and the regenerative cycle mode. The entire experiment began at 13:00 pm and continued until 16:25 pm. The average ambient temperature during the experiment was $25^{\circ} \mathrm{C}$ and the ambient pressure was $0.1 \mathrm{MPa}$. In the initial stage, only the heat collecting cycle is run, and the inlet and outlet parameters of the heat transfer oil in the collector and the corresponding solar radiation intensity data are collected under different heat transfer oil flow rates, and the solar collector is under different heat transfer oil flow rates. The heat collection efficiency was measured. The organic working fluid cycle starts from $15: 05$, the initial time runs in the basic cycle mode, then starts switching to the regenerative cycle mode at 15:25 and continues until the end of the experiment.

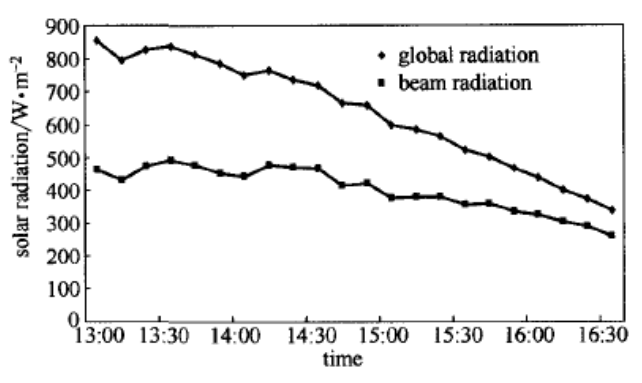

Fig.2 Measured solar radiation variation

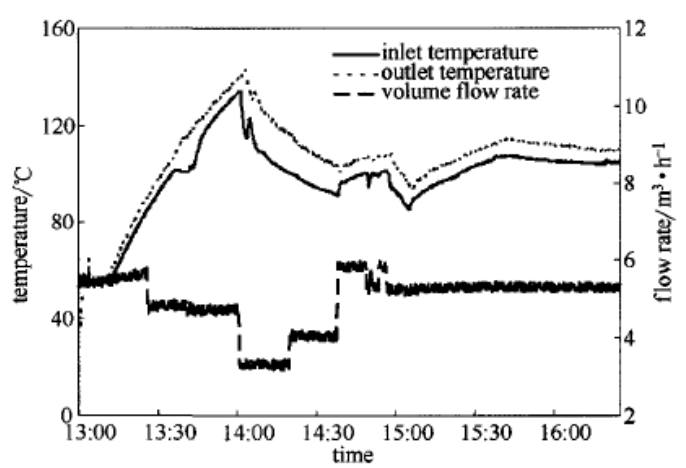

Fig.3 Heat exchanger oil temperature and heat transfer oil flow change at the collector inlet

Fig. 2 shows the measured solar radiation intensity as a function of time. During the test period, both the total solar radiation intensity and the direct radiation intensity gradually decrease over time, and the direct radiation intensity is maintained at $400 \mathrm{~W} \cdot \mathrm{m}-2$ for a period of time. Fig. 3 shows the changes in the temperature of the heat transfer oil and the flow rate of the heat transfer oil at the inlet and outlet of the collector during the whole experiment. From 13:00 to 15:00, the heat transfer oil only circulates in the heat collecting system. At this stage, the direct radiation intensity is basically maintained at $400 \mathrm{~W} \cdot \mathrm{m}-2$, and the heat transfer oil flow has several adjustment changes. During this time, the heat transfer oil absorbs heat in the collector and the temperature rises. At lower solar radiation intensity, the heat transfer temperature can be raised to $150{ }^{\circ} \mathrm{C}$. At 14:00, in order to control the temperature of the heat transfer oil at the collector outlet, the heat transfer oil cooler was turned on, and the heat transfer oil cooler was turned off at around 14:40. It can be clearly seen from the figure that the temperature difference of the heat transfer oil at the inlet and outlet of the collector is mainly affected by the flow rate of the heat transfer oil and the radiation conditions. Under the condition that the direct radiation intensity of the sun is constant, the temperature difference of the heat transfer oil at the inlet and outlet of the collector increases with the decrease of the flow rate of the heat transfer oil. Comparing the data before and after 15:00, it can be concluded that although the flow rate of the heat transfer oil is reduced, the temperature difference between the inlet and outlet does not increase, which is mainly due to the decrease of the direct radiation intensity after 15:00.

The power generation efficiency of the orc generator set is shown in Fig. 4. It can be seen from the figure that 
in the power generation system, the test results show that the inlet temperature of the expander is $\mathrm{T} 1=138.5^{\circ} \mathrm{C}$, and the corresponding enthalpy value $\mathrm{H} 1=468.48 \mathrm{~kJ} / \mathrm{kg} .{ }^{\circ} \mathrm{C}$, the inlet temperature of the refrigerant pump $\mathrm{T} 1=35.6^{\circ} \mathrm{C}$, the corresponding enthalpy value $\mathrm{H} 2=248 \mathrm{~kJ} / \mathrm{kg}$. ${ }^{\circ} \mathrm{C}$, refrigerant flow rate $q=611 \mathrm{~L} / \mathrm{h}$, density $\rho=1.43 \mathrm{~kg} / \mathrm{L}$, so the output heat of evaporation heat absorption to the expander is obtained $\mathrm{P} 1=\mathrm{q}^{*} \mathrm{\rho} / 3600 *(\mathrm{H} 1-\mathrm{H} 2)=53.5 \mathrm{~kW}$, the detected power generation $\mathrm{P}=4.78 \mathrm{~kW}$, power generation efficiency $\eta=\mathrm{P} / \mathrm{P} 1 * 100 \%=8.9 \%$.

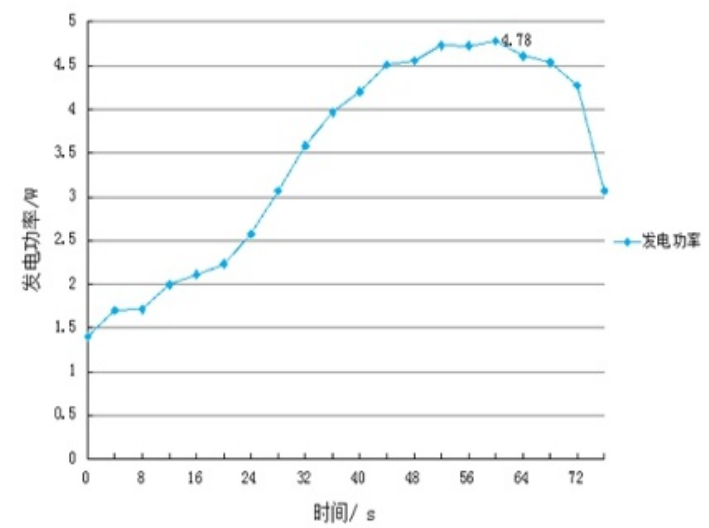

Fig. 4 ORC generator set power generation performance test

\section{Conclusion}

(1) A solar-driven organic Rankine cycle system test bench was established, and a series of experiments were carried out to obtain stable operation data of the system under different cycle modes, which can provide guidance for practical projects.

(2) Under medium radiation conditions, the solar collectors used in the experimental system can still effectively collect solar energy. Under $400 \mathrm{~W} \cdot \mathrm{m}-2$ solar direct radiation, the heat collection temperature can reach $150{ }^{\circ} \mathrm{C}$, and the measured heat collection efficiency is up to About $60 \%$.

(3) The use of the regenerator can effectively improve the cycle efficiency of the system under certain conditions. In the case where the superheat of the expander of the expander is large in the experiment, the cycle efficiency in the recovered heat cycle mode is $6 \%$ higher than that in the basic cycle mode.

\section{REFERENCES}

1. Hung $\mathrm{T}$ C, Shai $\mathrm{T}$ Y Wang $\mathrm{S}$ K. A review of organic Rankine cycles (ORCs)for the recovery of low-grade waste heat[J]. Energy, 1997, 22(7): 661667

2. Nishith B Desai, San tan u Bandyopadhyay. Process integration of organic Rankine cycle[J]. Energy, 2009, 34: 1674-1686

3. Manolakos D, Papadakis G Mohamed E S, et a1. Design of an autonomous low-temperature solar
Rankine cycle system for reverse osmosis desalination [J]. Desalination, 2005, 183:73-80

4. Nguyen V M, Doherty P S, Rifat S B. Development of a prototype low-temperature Rankine cycle electricity generation system[J]. AppliedThermalEngineering, 2001, 21:169-181

5. Christoph W ieland, Dominik $M$ einel, Hartmut Spliethof. Exergoeconomic comparison of ORC concepts at diferent scales/2nd International Seminar on ORC Power Systems(ASME ORC2013)[C]. The Netherland, 2013

6. Sotirios Karellas, Konstantinos Braimakis . Hybrid biomass and solar energy-based cogeneration an $\mathrm{d}$ trigeneration systems combining ore- $\mathrm{vcc}(\mathrm{C}) / / 2 \mathrm{nd}$ International Seminar on ORC Power Systems(ASME ORC2013)[C]. The Netherlands, 2013

7. $\mathrm{W}$ ang $\mathrm{X}$ D, Zhao L, Wan g J L. Experimental investigation on the low-temperature solar Rankine cycle system using R245fa[J]. Energy Conversion andManagement, 2011, 52: 946-952

8. Li Jing, Pei Gang, Ji Jie. Analysis of key factors in low--temperature solar-thermal--electric power generation with organic Rankine cycle[J]. CIESC Journal, 2009, 60(4): 826-832

9. Liu Linding . Research on the single screw expander and organic Rankine cycle system [D]. Beijing: Beijing University of Technology,2010

10. Song Jianzhong, Zhang Xiaosong . A theoretical study on low'temperature solar thermal power cycle[J]. Journal of CentralSouth University : Science and Technology, 2012, 43(10): 4075-4080 